\title{
Towards a better understanding of Artemisia vulgaris: botany, phytochemistry, pharmacological and biotechnological potential
}

\begin{abstract}
Artemisia vulgaris is one of the important medicinal plant species of the genus Artemisia, which is usually known for its volatile oils. The genus Artemisia has become the subject of great interest due to its chemical and biological diversity as well as the discovery and isolation of promising anti-malarial drug artemisinin. A. vulgaris has a long history in treatment of human ailments by medicinal plants in various parts of the world. This medicinal plant possesses a broad spectrum of therapeutic properties including: anti-malarial, antiinflammatory, anti-hypertensive, anti-oxidant, anti-tumoral, immunomodulatory, hepatoprotective, anti-spasmodic and anti-septic. These activities are mainly attributed to the presence of various classes of secondary metabolites, including flavonoids, sesquiterpene lactones, coumarins, acetylenes, phenolic acids, organic acids, mono- and sesquiterpenes. Studies related to A. vulgaris morphology, anatomy and phytochemistry has gained a significant interest for better understanding of production and accumulation of therapeutic compounds in this species. Recently, phytochemical and pharmacological investigations have corroborated the therapeutic potential of bioactive compounds of A. vulgaris. These findings provided further evidence for gaining deeper insight into the identification and isolation of novel compounds, which act as alternative sources of anti-malarial drugs in a cost-effective manner. Considering the rising demand and various medical applications of A. vulgaris, this review highlights the recent reports on the chemistry, biological activities and biotechnological interventions for controlled and continuous production of bioactive compounds from this plant species.
\end{abstract}

Keyword: Asteraceae; Artemisinin; Essential oils; Mugwort; Pharmacology 